\title{
Mid-Infrared Variability in Binary Brown Dwarfs
}

\author{
Michael F. Sterzik ${ }^{1}$, Gael Chauvin ${ }^{2}$, Kerstin Geißler ${ }^{1,3}$ \\ and Eric Pantin ${ }^{4}$ \\ ${ }^{1}$ European Southern Observatory, Casilla 19001, Santiago 19, Chile \\ email: msterzik@eso.org \\ ${ }^{2}$ Laboratoire d' Astrophysique, Observatoire de Grenoble, 38041 Grenoble Cedex 9, France \\ ${ }^{3}$ Max-Planck-Institut for Astronomy, Königstuhl 17, 69117 Heidelberg, Germany \\ ${ }^{4}$ CEA/Saclay, DSM/DAPNIA/Service d' Astrophysique, 91191 Gif-sur-Yvette, France
}

\begin{abstract}
We have spatially resolved several nearby binary brown dwarfs and obtained midinfrared photometry with VISIR at the VLT. In particular, we have monitored $\varepsilon$ Indi B and HD 130948 in several narrow-band MIR filters. The $10.5 \mu \mathrm{m}$ band is a probe to constrain nonequilibrium chemistry in the atmosphere of cool brown dwarfs.
\end{abstract}

Keywords. stars: low-mass, brown dwarfs; binaries: close

\section{Ground-based MIR measurements}

Ground-based mid-IR imaging of binary brown dwarf systems with sub-arcsecond spatial resolution can complement high sensitivity, but low-spatial resolution space-based photometry as obtained e.g. with Spitzer. The spatially resolved photometry of the close (seperation 0.7") brown dwarf binary $\varepsilon$ Indi $\mathrm{Ba}$ and $\mathrm{Bb}$ (Sterzik, Pantin, Hartung et al. 2005) and of three other brown dwarfs in binary systems, GJ 229 B (separation 7.8"), HD 130948 B (separation 2.6", B itself a L4 binary with a separation of 0.1 ") and HR 7329 B (separation 4.2") allows to constrain atmospheric models of ultra-cool brown dwarfs of various ages and metallicities (Geißler, Chauvin and Sterzik 2008). On-source integration times of about one hour in the $8.6 \mu \mathrm{m}, 10.5 \mu \mathrm{m}$ and $11.3 \mu \mathrm{m}$ bandpasses yield $3 \sigma$ detection sensitivities of less then $1-2 \mathrm{mJy}$ for point sources. In case of the HD $130948 \mathrm{~B}$, we have noticed a flux variation of at least $1.7 \pm 0.6 \mathrm{mJy}$ within 48 hours in the $10.5 \mu \mathrm{m}$ bandpass and could not explain it through insufficient sensitivity during one epoch of observations. Therefore we conducted time-series measurements in order to probe potential variability. In particular, significant variations in the $10.5 \mu \mathrm{m}$ band may be expected in the atmospheres of brown dwarfs at the $\mathrm{L} / \mathrm{T}$ transition in case non-equilibrium chemistry affecting the $\mathrm{CO}, \mathrm{CH}_{4}$ and $\mathrm{NH}_{3}$ abundances is important (Hubeny and Burrows 2007). While in the case of HD 130948 B the likelihood of variability is small (Geißler et al. 2009), $\varepsilon$ Indi Ba (a L/T transition object) may be variable in $10.5 \mu \mathrm{m}$.

\section{References}

Geißler, K., Chauvin, G., \& Sterzik, M. F. 2008, A\&A 480, 193

Geißler, K., Sterzik, M. F., Chauvin, G., \& Pantin, E. 2009, in: E. Stempels (ed.), Cool Stars, Stellar Systems and the Sun, 15th Cambridge Workshop (AIP), p. 521

Hubeny, I. \& Burrows, A. 2007, ApJ 669, 1248

Sterzik, M. F., Pantin, E., Hartung, M., Huelamo, N., Kaufer, A., Käufl, H. U., Melo, C., Nürnberger, D., Siebenmorgen, R., \& Smette, A. 2005, A\& A 436, L39 